\title{
ROBUST STABILITY AND PERFORMANCE VIA FIXED-ORDER DYNAMIC COMPENSATION*
}

\author{
DENNIS S. BERNSTEIN $\dagger$
}

\begin{abstract}
Two robust control-design problems are considered. The Robust Stabilization Problem involves deterministically modeled, bounded but unknown, time-varying parameter variations, while the Robust Performance Problem includes, in addition, a quadratic performance criterion averaged over stochastic disturbances and maximized over the admissible parameter variations. For both problems the design goal is a fixed-order (i.e., reduced- or full-order) dynamic (strictly proper) feedback compensator. A sufficient condition for solving the Robust Stabilization Problem is given by means of a quadratic Lyapunov function parameterized by the compensator gains. For the Robust Performance Problem the Lyapunov function provides an upper bound for the closed-loop performance. This leads to consideration of the Auxiliary Minimization Problem: Minimize the performance bound over the class of fixed-order controllers subject to the Lyapunov-function constraint. Necessary conditions for optimality in the auxiliary problem thus serve as sufficient conditions for robust stability and performance in the original problem. Two particular bounds are considered for constructing the quadratic Lyapunov function. The first corresponds to a right shift/multiplicative white noise model, while the second was suggested by recent work of Petersen and Hollot. The main result is an extended version of the optimal projection equations for fixed-order dynamic compensation whose solutions are guaranteed to provide both robust stability and robust performance.
\end{abstract}

Key words. robust control, stability, performance, dynamic compensation, Lyapunov bounds

AMS(MOS) subject classification. 93

1. Introduction. Although considerable effort has been devoted to frequencydomain robust-control design methods [1]-[10], there remain open questions concerning stability with respect to real-valued, structured plant parameter variations [11]-[13]. Specifically, it is shown in [11]-[13] that classical gain and phase margin specifications can be satisfied, while sensitivity to structured plant parameter variations can be arbitrarily large. From a time-domain point of view, the parametric robustness problem has been widely studied using Lyapunov's second method as the principal technique [14]-[28].

In this paper we develop an approach to control design that provides sufficient conditions for robust stability and performance over a prescribed range of time-varying structured plant parameter variations by means of a feedback law in the form of a fixed-order (i.e., reduced- or full-order) dynamic (strictly proper) compensator. The approach is based upon the merging of two techniques, namely, the guaranteed cost control approach to robust performance [14], [17] and the optimal projection approach to quadratically optimal fixed-order dynamic compensation [29], [30]. One of our goals is to obtain robust output-feedback compensators rather than full-state-feedback controllers. Also, since we wish to account for real-time computational burden in implementating the controller, we impose a constraint on the dimension (i.e., order) of the dynamic compensator. This approach thus generalizes standard LQG theory, which yields full-order output-feedback controllers for systems without parameter uncertainty. We note that our approach is constructive in the sense that, upon satisfaction of the sufficient conditions, the feedback gains required for implementing the robust feedback controller are explicitly synthesized. Existential issues are also addressed

* Received by the editors June 16, 1986; accepted for publication (in revised form) May 10, 1988. This research was supported in part by Air Force Office of Scientific Research contract F49620-86-C-0002.

† Harris Corporation, Melbourne, Florida 32901. 
herein, although to a lesser extent. For further background see [29], [30]. For extensions to nonstrictly proper controllers see [31], and for extensions to $H_{\infty}$ control see [32].

To explain the rationale behind the development we briefly describe the main elements of the approach. The following discussion is intended to be descriptive; precise conditions appear in the main body of the paper.

1.1. Robust Stability Problem. For a nominal linear time-invariant $(A, B, C)$ system we consider deterministically modeled bounded but otherwise unknown Lebesgue measurable time-varying parameter variations of the form

$$
A+\sum_{i=1}^{p} \hat{\sigma}_{i}(t) A_{i}, \quad B+\sum_{i=1}^{p} \hat{\sigma}_{i}(t) B_{i}, \quad C+\sum_{i=1}^{p} \hat{\sigma}_{i}(t) C_{i} .
$$

The nominal matrices $A, B, C$ and the perturbation matrices $A_{i}, B_{i}, C_{i}$ denoting the structure of the parametric uncertainty are assumed known, while the time-varying uncertain parameters $\hat{\sigma}_{i}(t)$ are assumed only to satisfy the bounds

$$
\left|\hat{\sigma}_{i}(t)\right| \leqq \delta_{i}, \quad i=1, \cdots, p, \quad t \in[0, \infty) .
$$

The form of (1.1) permits an arbitrary number of uncertain parameters with arbitrary linear structure. Although we do not require matching conditions as in [21], the linear structure of (1.1) is more restrictive than the functional form $A(q(t))$ used in [21]. It is this structure that we exploit to obtain sufficiency conditions. Note also that the representation (1.1) is independent of state space basis, since replacing $A$ by $S A S^{-1}$ corresponds to replacing $A_{i}$ by $S A_{i} S^{-1}$. As will be seen, our robustness bounds and optimality conditions are also basis independent. Also, scaling techniques [6], [7] will not play a role here. Finally, we note that because of the time-varying nature of the uncertain perturbations (1.1) it is virtually impossible to determine the actual stability region of a given design by means of empirical methods.

1.2. Quadratic Lyapunov function. As a sufficient condition for characterizing solutions of the Robust Stability Problem we consider a closed-loop quadratic Lyapunov function $V(\tilde{x})=\tilde{x}^{T} \mathscr{P} \tilde{x}$, where the matrix $\mathscr{P}$ satisfies

$$
0=\tilde{A}^{T} \mathscr{P}+\mathscr{P} \tilde{A}+\Omega\left(\mathscr{P}, B_{c}, C_{c}\right)
$$

and the function $\Omega$ is a bound satisfying

$$
\sum_{i=1}^{p} \sigma_{i}\left(\tilde{A}_{i}^{T} \mathscr{P}+\mathscr{P} \tilde{A}_{i}\right)<\Omega\left(\mathscr{P}, B_{c}, C_{c}\right)
$$

over the parameter range

$$
\left|\sigma_{i}\right| \leqq \delta_{i}, \quad i=1, \cdots, p .
$$

Note that the constant $\sigma_{i}$ in (1.4) and (1.5) plays the role of $\hat{\sigma}_{i}(t)$, i.e., $t$ is "frozen" in (1.4) and (1.5). In (1.3) and (1.4), $\tilde{A}$ and $\tilde{A}_{i}$ denote the closed-loop dynamics and closed-loop parameter-uncertainty matrices given by

$$
\tilde{A}=\left[\begin{array}{cc}
A & B C_{c} \\
B_{c} C & A_{c}
\end{array}\right], \quad \tilde{A}_{i}=\left[\begin{array}{cc}
A_{i} & B_{i} C_{c} \\
B_{c} C_{i} & 0
\end{array}\right] .
$$

Since $\tilde{A}_{i}$ is independent of $A_{c}, \Omega$ depends only on $B_{c}$ and $C_{c}$. As discussed later in this section, (1.4) is automatically satisfied by construction of the function $\Omega$. Furthermore, the existence of a solution $\mathscr{P}$ to (1.3) need not be verified directly, but rather is a result of numerically solving the optimality conditions discussed below. 
1.3. Robust Performance Problem. In addition to the deterministic parameter uncertainty model (1.1), (1.2), the Robust Performance Problem includes stochastic plant disturbances and measurement noise with performance measured by means of the quadratic functional

$$
\tilde{J}(t)=x^{T}(t) R_{1} x(t)+2 x^{T}(t) R_{12} u(t)+u^{T}(t) R_{2} u(t) .
$$

To obtain a steady-state design problem we (1) average $\tilde{J}(t)$ over the disturbance and measurement noise statistics; (2) pass to the steady-state limit; and (3) maximize over the class of parameter uncertainties. Hence the performance of a given controller $\left(A_{c}, B_{c}, C_{c}\right)$ is given by

$$
J\left(A_{c}, B_{c}, C_{c}\right)=\sup _{\hat{\sigma}(\cdot)} \limsup _{t \rightarrow \infty} \mathbb{E}[\tilde{J}(t)] .
$$

The use of "lim sup" is a technicality that accounts for cases in which the steady-state limit may not exist. Note that although (1.8) is an averaging criterion over the disturbances as in LQG theory, it is also a worst-case measure over the uncertain parameters. Thus (1.8) is a hybrid criterion in the sense that is stochastic in the disturbance space (i.e., external uncertainties) and deterministic in the parameter space (i.e., internal uncertainties). By "internal uncertainties" we have in mind quantities such as mass, damping, or stiffness; by "external uncertainties" we are referring to phenomena such as turbulent flow for which only power spectrum statistics may be available. No claim is made, however, with regard to the universal validity of such a mathematical uncertainty model. In particular applications, uncertainty models that are either wholly deterministic or wholly stochastic may be more appropriate. In general, our setting appears to be consistent with the available literature (see [1]-[28]).

1.4. Performance bound. To obtain a tractable design problem, we use the matrix $\mathscr{P}$ to bound the performance of each controller solving the Robust Stability Problem. Specifically, by assuming in addition to (1.4) that

$$
\sum_{i=1}^{p} \sigma_{i}\left(\tilde{A}_{i}^{T} \mathscr{P}+\mathscr{P} \tilde{A}_{i}\right)+\tilde{R} \leqq \Omega\left(\mathscr{P}, B_{c}, C_{c}\right),
$$

it follows that

$$
J\left(A_{c}, B_{c}, C_{c}\right) \leqq \operatorname{tr} \mathscr{P} \tilde{V} .
$$

In (1.9) and (1.10) $\tilde{R}$ and $\tilde{V}$ denote closed-loop weighting and disturbance intensity matrices. The idea of bounding the performance by means of a Lyapunov function is the basis for guaranteed cost control [14], [17]. ${ }^{1}$

1.5. Construction of the Lyapunov function. So far the Lyapunov function has only been abstractly characterized by means of (1.3) and (1.4). To obtain a useful design theory $\Omega$ is now given a concrete form. Specifically, to satisfy (1.9) it is assumed that

$$
\Omega\left(\mathscr{P}, B_{c}, C_{c}\right)=\sum_{i=1}^{p} \Lambda_{i}\left(\mathscr{P}, B_{c}, C_{c}\right)+\tilde{R},
$$

where, for each $i$, the $\Lambda_{i}$ are chosen such that

$$
\sigma_{i}\left(\tilde{A}_{i}^{T} \mathscr{P}+\mathscr{P} \tilde{A}_{i}\right) \leqq \Lambda_{i}\left(\mathscr{P}, B_{c}, C_{c}\right), \quad\left|\sigma_{i}\right| \leqq \delta_{i} .
$$

\footnotetext{
${ }^{1}$ It is also interesting to note that in Hamilton-Jacobi-Bellman sufficiency theory the performance functional is expressed in terms of a value function that also serves as a Lyapunov function for the closed-loop system. These connections will be explored in a future paper.
} 
Note that (1.12) implies that (1.4) holds with $\Omega$ given by (1.11). Since $\tilde{A}_{i}$ depends on $B_{c}$ and $C_{c}$, the bound $\Lambda_{i}$ will be constructed to be gain-invariant, that is, so that (1.12) holds for all $B_{c}$ and $C_{c}$. Thus no difficulty will arise from the fact that the controller gains are yet to be determined by optimality considerations.

It should be noted that the bounding in (1.12) is defined in the sense of the cone of nonnegative definite matrices. Since this is only a partial ordering and not a total ordering, a least upper bound (i.e., a "sharpest" bound) does not exist in general and the conservatism of the inequality in (1.12) cannot be quantified by a scalar measure. Hence, $\Lambda_{i}$ satisfying (1.12) is not necessarily unique and two particular choices of $\Lambda_{i}$ are developed in this paper. Since we shall utilize first-order necessary conditions for optimality, we confine our consideration to bounds that are differentiable. The first choice of $\Lambda_{i}$ satisfying (1.12) is given by the linear (in $\mathscr{P}$ ) function

$$
\Lambda_{i}\left(\mathscr{P}, B_{c}, C_{c}\right)=\delta_{i}\left(\alpha_{i} \mathscr{P}+\alpha_{i}^{-1} \tilde{A}_{i}^{T} \mathscr{P} \tilde{A}_{i}\right),
$$

where $\alpha_{i}$ is an arbitrary positive number. As shown in [33], the bound (1.13) can be viewed as arising from a stochastic optimal control problem with exponentially weighted cost and state-, control- and measurement-dependent white noise. The stochastic multiplicative white noise model serves only as an interpretation, however, and need not be viewed as having physical significance. A similar bound is used in [28].

The second choice for $\Lambda_{i}$ satisfying $(1,12)$ is given by the quadratic (in $\mathscr{P}$ ) function

$$
\Lambda_{i}\left(\mathscr{P}, B_{c}, C_{c}\right)=\delta_{i}\left(\tilde{E}_{i}^{T} \tilde{E}_{i}+\mathscr{P} \tilde{D}_{i} \tilde{D}_{i}^{T} \mathscr{P}\right),
$$

where $\tilde{D}_{i}, \tilde{E}_{i}$ denote an arbitrary factorization of $\tilde{A}_{i}$ of the form

$$
\tilde{A}_{i}=\tilde{D}_{i} \tilde{E}_{i} .
$$

The bound (1.14) was used in [26] for full-state feedback with rank 1 uncertainties. Note that using congruence transformations shows that both bounds (1.13) and (1.14) are basis independent; that is, replacing $\tilde{A}_{i}$ by $\tilde{S} \tilde{A}_{i} \tilde{S}^{-1}$ leads to replacing $\mathscr{P}$ by $\tilde{S}^{-T} \mathscr{P} \tilde{S}^{-1}$.

1.6. Auxiliary Minimization Problem. The next step in our development for robust performance is the following. Inasmuch as the performance of a robustly stabilizing controller is bounded via (1.10) over the given range of parameter variations, it is desirable to minimize the upper bound

$$
\mathscr{J}\left(\mathscr{P}, A_{c}, B_{c}, C_{c}\right) \triangleq \operatorname{tr} \mathscr{P} \tilde{V}
$$

subject to the constraint (1.3). This is referred to as the Auxiliary Minimization Problem. For a given choice (1.13) or (1.14) of $\Lambda_{i}$ for each $i$, a solution of the Auxiliary Minimization Problem provides a controller whose steady-state performance is guaranteed to remain below the bound (1.16) over the range of parameter variations, hence guaranteeing robust performance. Since the Auxiliary Minimization Problem is a smooth mathematical programming problem, a minimum always exists on compact sets. To actually characterize extremals of the Auxiliary Minimization Problem we proceed by deriving first-order necessary conditions. Because these necessary conditions are derived for the Auxiliary Minimization Problem, they effectively serve as sufficient conditions for robustness in the original problem.

It should be noted that the guaranteed cost control approach developed in [14] does not permit this line of development since $\Lambda_{i}$ is given by

$$
\Lambda_{i}\left(\mathscr{P}, B_{c}, C_{c}\right)=\delta_{i}\left|\tilde{A}_{i}^{T} \mathscr{P}+\mathscr{P} \tilde{A}_{i}\right|,
$$


where $|\cdot|$ denotes the matrix obtained by replacing each eigenvalue by its absolute value. Since this bound is not differentiable with respect to the controller gains, first-order necessary conditions cannot be used.

1.7. The optimality conditions: full-order case. For the full-order case, i.e., when the order of the controller is equal to the order of the plant, the first-order necessary conditions can be derived in a form that is a direct generalization of the pair of separated Riccati equations of LQG theory. Specifically, the necessary conditions comprise a coupled system of four algebraic matrix equations including a pair of modified Riccati equations and a pair of Lyapunov equations. For plant models involving multiplicative white noise these equations have been studied in [34]-[36]. This form of the equations thus essentially corresponds to choosing bound (1.13).

1.8. The optimality conditions: reduced-order case. For design flexibility we also consider controllers of arbitrary reduced dimension. For the linear-quadratic problem without parameter uncertainty, the formulation of the necessary conditions given in [29] provides a generalization of LQG theory. Specifically, the optimal gains are characterized by a system of algebraic matrix equations consisting of a pair of modified Riccati equations and a pair of modified Lyapunov equations coupled by an oblique projection. When the order of the controller is equal to the order of the plant, the projection becomes the identity and the standard LQG result is recovered.

The outcome of the development above is a set of algebraic matrix equations that correspond to the necessary conditions for the Auxiliary Minimization Problem and hence to sufficient conditions for robust stability and performance. These necessary conditions characterize full- or reduced-order controllers with either choice of bounds (1.13) and (1.14) for each uncertain parameter. For control-system design, these equations can be used as follows. If a solution to the necessary conditions is obtained computationally and if certain definiteness conditions hold, then the explicitly synthesized controller (1) solves the Robust Stability Problem and (2) is guaranteed to provide robust performance bounded by $\operatorname{tr} \mathscr{P} \tilde{V}$ over the stipulated uncertainty range.

The applicability of these results is, of course, limited to plants that are nominally stabilizable via controllers of the given order. Indeed, in this case it has been shown [37] via topological degree theory that the optimality conditions for the case $\delta_{i}=0$, $i=1, \cdots, p$, possess at least one stabilizing solution. For the parameter uncertainty problem, i.e., $\delta_{i}>0$, it follows from continuity properties that a solution also exists for sufficiently small $\delta_{i}$. The actual range of uncertainty that can be stabilized and the tightness of the performance bound depend on the conservatism of our bounds. As will be seen from a numerical example, our bounds are not generally sharp. This is not unexpected, however, due to both the sense of the partial ordering employed in (1.12) and the fact that our choice of gain-invariant bounds permits a one-step, noniterative synthesis (rather than analysis) procedure. It should be noted that necessary and sufficient conditions for robust analysis of a block-structured class of uncertainties are obtainable using the $\mu$-function [6]. This block structure, however, does not appear to include either the linear uncertainty model (1.1) or the matched uncertainty model of [21] as special cases.

In the present paper we present results of an illustrative numerical study for a well-known example used in [2] to demonstrate the lack of gain margin for LQG controllers. This type of uncertainty is a special case of (1.1) obtained by taking $p=m$ and defining $B_{i}$ to be the matrix whose $i$ th column is the same as the $i$ th column of $B$, and zero otherwise. To obtain full-order, robustified controllers exhibiting performance/robustness tradeoffs, we use bound (1.13) for several values of $\delta_{i}$. To obtain 
these numerical results we used a straightforward iterative algorithm that requires only an LQG-type software package. The homotopy algorithm of [37] with appropriate extensions can also be used. Further descriptions of related algorithms and numerical results can be found in [38]-[40].

The development herein is self-contained, with the exception that the detailed derivation of the optimality conditions has been omitted. In specialized cases the derivation has been given previously. For the case of bound (1.13) only, a derivation using Kronecker products appears in [36]. Also, a derivation without parameter uncertainties has been given in [29] using Lagrange multipliers. Overall, the derivation involves considerable matrix manipulation. Since the detailed derivation does not appear to warrant the required space, we give an outline of the proof to assist the sufficiently motivated reader in reconstructing the details.

2. Notation and definitions. (Note that all matrices have real entries.)

$\mathbb{R}, \mathbb{R}^{r \times s}, \mathbb{R}^{r}, \mathbb{E}$

$$
\|\cdot\|
$$

$I_{r}, 0_{r \times s}, 0_{r}$

()$^{T},()^{-1},()^{-T}$

$\operatorname{tr}$

$\oplus, \otimes$

$\mathbb{S}^{r}$

$\mathbb{N}^{r}$

$\mathbb{P}^{r}$

$Z_{1} \geqq Z_{2}$

$Z_{1}>Z_{2}$

asymptotically stable matrix

$n, m, l, p, n_{c}, n_{i}, m_{i}$

$\tilde{n}, \tilde{n}_{i}$

$x, u, y, x_{c}$

$A, A_{i} ; B, B_{i} ; C, C_{i}$

$A_{c}, B_{c}, C_{c}$

$\tilde{A}, \tilde{A}_{i}$

$\delta_{i}$

$\Delta$

$\sigma_{i}$

$\sigma$

$\hat{\sigma}_{i}(\cdot)$

$\hat{\sigma}(\cdot)$

$L_{\infty}([0, \infty), \Delta)$

$\alpha_{i}$

$D_{i}, E_{i}, H_{i}, K_{i}$

$\tilde{D}_{i}, \tilde{E}_{i}$

$\sum^{\prime}, \sum^{\prime \prime}$

$R_{1}$ real numbers, $r \times s$ real matrices, $\mathbb{R}^{r \times 1}$,

expectation

Euclidean vector norm

$r \times r$ identity matrix, $r \times s$ zero matrix, $0_{r \times r}$

transpose, inverse, inverse transpose

trace

Kronecker sum, Kronecker product [41]

$r \times r$ symmetric matrices

$r \times r$ symmetric nonnegative-definite matrices

$r \times r$ symmetric positive-definite matrices

$Z_{1}-Z_{2} \in \mathbb{N}^{r}, Z_{1}, Z_{2} \in \mathbb{S}^{r}$

$Z_{1}-Z_{2} \in \mathbb{P}^{r}, Z_{1}, Z_{2} \in \mathbb{S}^{r}$

matrix with eigenvalues in open left half-plane

positive integers, $i \in\{1, \cdots, p\}$

$n+n_{c}, n_{i}+m_{i}, i \in\{1, \cdots, p\}$

$n, m, l, n_{c}$-dimensional vectors

$n \times n$ matrices, $n \times m$ matrices, $l \times n$ matrices,

$i \in\{1, \cdots, p\}$

$n_{c} \times n_{c}, n_{c} \times l, m \times n_{c}$ matrices

$\left[\begin{array}{cc}A & B C_{c} \\ B_{c} C & A_{c}\end{array}\right],\left[\begin{array}{cc}A_{i} & B_{i} C_{c} \\ B_{c} C_{i} & 0\end{array}\right], i \in\{1, \cdots, p\}$

positive number, $i \in\{1, \cdots, p\}$

$\left[-\delta_{1}, \delta_{1}\right] \times \cdots \times\left[-\delta_{p}, \delta_{p}\right]$

real number, $i \in\{1, \cdots, p\}$

$\left(\sigma_{1}, \cdots, \sigma_{p}\right)$

Lebesgue measurable function on $[0, \infty), i \in$

$\{1, \cdots, p\}$

$\left(\hat{\sigma}_{1}(\cdot), \cdots, \hat{\sigma}_{p}(\cdot)\right)$

Lebesgue measurable functions on $[0, \infty)$ with values in $\Delta$

positive number, $i \in\{1, \cdots, p\}$

$n \times n_{i}, n_{i} \times n, n \times m_{i}, m_{i} \times m$ matrices,

$i \in\{1, \cdots, p\}$

$\tilde{n} \times \tilde{n}_{i}, \tilde{n}_{i} \times \tilde{n}$ matrices, $i \in\{1, \cdots, p\}$

see $\S 6$

state weighting matrix in $\mathbb{N}^{n}$ 


$\begin{array}{ll}R_{2} & \text { control weighting matrix in } \mathbb{P}^{m} \\ R_{12} & n \times m \text { cross weighting matrix such tha } \\ & R_{1}-R_{12} R_{2}^{-1} R_{12}^{T} \geqq 0 \\ \tilde{R} & {\left[\begin{array}{cc}R_{1} & R_{12} C_{c} \\ C_{c}^{T} R_{12}^{T} & C_{c}^{T} R_{2} C_{c}\end{array}\right]} \\ w_{1}(\cdot) & n \text {-dimensional white noise } \\ w_{2}(\cdot) & \text { l-dimensional white noise } \\ V_{1} & \text { intensity of } w_{1}(\cdot) \text { in } \mathbb{N}^{n} \\ V_{2} & \text { intensity of } w_{2}(\cdot) \text { in } \mathbb{P}^{l} \\ V_{12} & n \times l \text { cross-intensity of } w_{1}(\cdot), w_{2}(\cdot) \\ \tilde{V} & {\left[\begin{array}{cc}V_{1} & V_{12} B_{c}^{T} \\ B_{c} V_{12}^{T} & B_{c} V_{2} B_{c}^{T}\end{array}\right]}\end{array}$

3. Robust Stability and Robust Performance Problems. In this section we state the Robust Stability Problem and Robust Performance Problem along with related notation for later use.

3.1. Robust Stability Problem. For fixed $n_{c} \leqq n$, determine $\left(A_{c}, B_{c}, C_{c}\right) \in$ $\mathbb{R}^{n_{c} \times n_{c}} \times \mathbb{R}^{n_{c} \times 1} \times \mathbb{R}^{m \times n_{c}}$ such that the closed-loop system consisting of the $n$ th-order controlled plant

$$
\dot{x}(t)=\left(A+\sum_{i=1}^{p} \hat{\sigma}_{i}(t) A_{i}\right) x(t)+\left(B+\sum_{i=1}^{p} \hat{\sigma}_{i}(t) B_{i}\right) u(t) \quad \text { a.a. } t \in[0, \infty),
$$

measurements

$$
y(t)=\left(C+\sum_{i=1}^{p} \hat{\sigma}_{i}(t) C_{i}\right) x(t),
$$

and $n_{c}$ th-order dynamic compensator

$$
\begin{aligned}
& \dot{x}_{c}(t)=A_{c} x_{c}(t)+B_{c} y(t), \\
& u(t)=C_{c} x_{c}(t)
\end{aligned}
$$

are asymptotically stable ${ }^{2}$ for all $\hat{\sigma}(\cdot) \in L_{\infty}([0, \infty), \Delta)$.

3.2. Robust Performance Problem. For fixed $n_{c} \leqq n$, determine $\left(A_{c}, B_{c}, C_{c}\right) \in$ $\mathbb{R}^{n_{c} \times n_{c}} \times \mathbb{R}^{n_{c} \times l} \times \mathbb{R}^{m \times n_{c}}$ such that, for the closed-loop system consisting of the $n$ th-order controlled and disturbed plant

$$
\dot{x}(t)=\left(A+\sum_{i=1}^{p} \hat{\sigma}_{i}(t) A_{i}\right) x(t)+\left(B+\sum_{i=1}^{p} \hat{\sigma}_{i}(t) B_{i}\right) u(t)+w_{1}(t) \text { a.a. } t \in[0, \infty),
$$

noisy measurements

$$
y(t)=\left(C+\sum_{i=1}^{p} \hat{\sigma}_{i}(t) C_{i}\right) x(t)+w_{2}(t),
$$

and $n_{c}$ th-order dynamic compensator (3.3), (3.4), the performance criterion

$$
\begin{aligned}
& J\left(A_{c}, B_{c}, C_{c}\right) \\
& \quad \stackrel{\Delta}{=} \sup _{\hat{\sigma}(\cdot) \in L_{\infty}([0, \infty), \Delta)} \limsup _{t \rightarrow \infty} \mathbb{E}\left[x^{T}(t) R_{1} x(t)+2 x^{T}(t) R_{12} u(t)+u^{T}(t) R_{2} u(t)\right]
\end{aligned}
$$

is minimized.

\footnotetext{
${ }^{2}$ Asymptotic stability for a nonautonomous system is defined in the standard way (see, e.g., [42]).
} 
For each controller $\left(A_{c}, B_{c}, C_{c}\right)$ and parameter variation $\hat{\sigma}(\cdot) \in L_{\infty}([0, \infty), \Delta)$ the undisturbed closed-loop system (3.1)-(3.4) is given by

$$
\dot{\tilde{x}}(t)=\left(\tilde{A}+\sum_{i=1}^{p} \hat{\sigma}_{i}(t) \tilde{A}_{i}\right) \tilde{x}(t) \quad \text { a.a. } t \in[0, \infty),
$$

while the disturbed closed-loop system (3.3)-(3.6) is

$$
\dot{\tilde{x}}(t)=\left(\tilde{A}+\sum_{i=1}^{p} \hat{\sigma}_{i}(t) \tilde{A}_{i}\right) \tilde{x}(t)+\tilde{w}(t) \quad \text { a.a. } t \in[0, \infty) .
$$

Also (see, e.g., [43, p. 194]), let $\tilde{\Phi}:[0, \infty) \rightarrow \mathbb{R}^{\tilde{n} \times \tilde{n}}$ be the unique absolutely continuous solution to

$$
\begin{gathered}
\dot{\tilde{\Phi}}(t)=\left(\tilde{A}+\sum_{i=1}^{p} \hat{\sigma}_{i}(t) \tilde{A}_{i}\right) \tilde{\Phi}(t) \quad \text { a.a. } t \in[0, \infty), \\
\tilde{\Phi}(0)=I_{\tilde{n}},
\end{gathered}
$$

and recall that $\tilde{\Phi}^{-1}(\cdot)$ satisfies

$$
\frac{d}{d t} \tilde{\Phi}^{-1}(t)=-\tilde{\Phi}^{-1}(t)\left(\tilde{A}+\sum_{i=1}^{p} \hat{\sigma}_{i}(t) \tilde{A}_{i}\right) \quad \text { a.a. } t \in[0, \infty) .
$$

4. Sufficient conditions for robust stability and performance. For robust stability we characterize quadratic Lyapunov functions for the closed-loop system.

THEOREM 4.1. Let $\Omega: \mathbb{P}^{\tilde{n}} \times \mathbb{R}^{n_{c} \times l} \times \mathbb{R}^{m \times n_{c}} \rightarrow \mathbb{S}^{\tilde{n}}$ satisfy

$$
\begin{aligned}
& \sum_{i=1}^{p} \sigma_{i}\left(\tilde{A}_{i}^{T} \mathscr{P}+\mathscr{P} \tilde{A}_{i}\right)<\Omega\left(\mathscr{P}, B_{c}, C_{c}\right), \quad \sigma \in \Delta, \\
& \left(\mathscr{P}, B_{c}, C_{c}\right) \in \mathbb{P}^{\tilde{n}} \times \mathbb{R}^{n_{c} \times l} \times \mathbb{R}^{m \times n_{c}} .
\end{aligned}
$$

If, for some $\left(A_{c}, B_{c}, C_{c}\right) \in \mathbb{R}^{n_{c} \times n_{c}} \times \mathbb{R}^{n_{c} \times I} \times \mathbb{R}^{m \times n_{c}}$, there exists $\mathscr{P} \in \mathbb{P}^{\tilde{n}}$ satisfying

$$
0=\tilde{A}^{T} \mathscr{P}+\mathscr{P} \tilde{A}+\Omega\left(\mathscr{P}, B_{c}, C_{c}\right)
$$

then $\left(A_{c}, B_{c}, C_{c}\right)$ solves the Robust Stability Problem.

Proof. Define the Lyapunov function

$$
V(\tilde{x}) \triangleq \tilde{x}^{T} \mathscr{P} \tilde{x}, \quad \tilde{x} \in \mathbb{R}^{\tilde{n}} .
$$

For almost all $t \in[0, \infty)$ and $\tilde{x}(t)$ satisfying (3.8), it follows from (4.2) that

$$
\begin{aligned}
\dot{V}(\tilde{x}(t)) & =\dot{x}^{T}(t) \mathscr{P} \tilde{x}(t)+\tilde{x}^{T}(t) \mathscr{P} \dot{x}(t) \\
& =\tilde{x}^{T}(t)\left[\left(\tilde{A}+\sum_{i=1}^{p} \hat{\sigma}_{i}(t) \tilde{A}_{i}\right)^{T} \mathscr{P}+\mathscr{P}\left(\tilde{A}+\sum_{i=1}^{p} \hat{\sigma}_{i}(t) \tilde{A}_{i}\right)\right] \tilde{x}(t) \\
& =\tilde{x}^{T}(t)\left[\sum_{i=1}^{p} \hat{\sigma}_{i}(t)\left(\tilde{A}_{i}^{T} \mathscr{P}+\mathscr{P} \tilde{A}_{i}\right)-\Omega\left(\mathscr{P}, B_{c}, C_{c}\right)\right] \tilde{x}(t) .
\end{aligned}
$$

Since $\hat{\sigma}(t) \in \Delta$, almost all $t \in[0, \infty)$, it follows from (4.1) that there exists $\gamma>0$ such that $\dot{V}(\tilde{x}(t)) \leqq-\gamma\|\tilde{x}(t)\|^{2}$, almost all $t \in[0, \infty)$.

Remark 4.1. If $\left(A_{c}, B_{c}, C_{c}\right)$ solves the Robust Stability Problem, then

$$
\lim _{t \rightarrow \infty} \tilde{\Phi}(t)=0, \quad \hat{\sigma}(\cdot) \in L_{\infty}([0, \infty), \Delta) \text {. }
$$

Remark 4.2. As will be seen, the bound (4.1) will be guaranteed for all $\mathscr{P}, B_{c}, C_{c}$ by suitable construction of the function $\Omega$. In addition, the existence of a solution $\mathscr{P}$ to (4.2) need not be verified in practice. Rather, (4.2) is a result of numerically solving the necessary conditions for the Auxiliary Minimization Problem given in Theorem 6.1. 
For the Robust Performance Problem the cost can be expressed in terms of the closed-loop second-moment matrix.

PROPOSITION 4.1. For $\left(A_{c}, B_{c}, C_{c}\right) \in \mathbb{R}^{n_{c} \times n_{c}} \times \mathbb{R}^{n_{c} \times l} \times \mathbb{R}^{m \times n_{c}}$ and $\hat{\sigma}(\cdot) \in L_{\infty}([0, \infty), \Delta)$ the second-moment matrix

$$
\tilde{Q}(t) \triangleq \mathbb{E}\left[\tilde{x}(t) \tilde{x}^{T}(t)\right], \quad t \in[0, \infty),
$$

satisfies

$$
\dot{\tilde{Q}}(t)=\left(\tilde{A}+\sum_{i=1}^{p} \hat{\sigma}_{i}(t) \tilde{A}_{i}\right) \tilde{Q}(t)+\tilde{Q}(t)\left(\tilde{A}+\sum_{i=1}^{p} \hat{\sigma}_{i}(t) \tilde{A}_{i}\right)^{T}+\tilde{V} \quad \text { a.a. } t \in[0, \infty),
$$

or, equivalently,

$$
\tilde{Q}(t)=\tilde{\Phi}(t) \tilde{Q}(0) \tilde{\Phi}^{T}(t)+\int_{0}^{t} \tilde{\Phi}(t) \tilde{\Phi}^{-1}(s) \tilde{V} \tilde{\Phi}^{-T}(s) \tilde{\Phi}^{T}(t) d s, \quad t \in[0, \infty)
$$

Furthermore,

$$
J\left(A_{c}, B_{c}, C_{c}\right)=\sup _{\hat{\sigma}(\cdot) \in L_{\infty}([0, \infty), \Delta)} \limsup _{t \rightarrow \infty} \operatorname{tr} \tilde{Q}(t) \tilde{R}
$$

or, equivalently,

$$
\begin{aligned}
J\left(A_{c}, B_{c}, C_{c}\right) \triangleq & \sup _{\hat{\sigma}(\cdot) \in L_{\infty}([0, \infty), \Delta)} \limsup _{t \rightarrow \infty} \operatorname{tr}\left[\tilde{\Phi}(t) \tilde{Q}(0) \tilde{\Phi}^{T}(t) \tilde{R}\right. \\
& \left.+\int_{0}^{t} \tilde{\Phi}(t) \tilde{\Phi}^{-1}(s) \tilde{V} \tilde{\Phi}^{-T}(s) \tilde{\Phi}^{T}(t) d s \tilde{R}\right] .
\end{aligned}
$$

Proof. The second-moment equation (4.5) is a direct consequence of the Itô differential rule (see [44, p. 142]), while (4.6) follows by direct verification. Finally, (4.7) is immediate.

We now obtain an upper bound for $J$ in terms of the matrix $\mathscr{P}$. The following lemma is required.

LeMmA 4.1. Let $\Omega: \mathbb{P}^{\tilde{n}} \times \mathbb{R}^{n_{c} \times l} \times \mathbb{R}^{m \times n_{c}} \rightarrow \mathbb{S}^{\tilde{n}}$ and $\left(A_{c}, B_{c}, C_{c}\right) \in \mathbb{R}^{n_{c} \times n_{c}} \times \mathbb{R}^{n_{c} \times l} \times$ $\mathbb{R}^{m \times n_{c}}$ be given. Then $\mathscr{P} \in \mathbb{P}^{\tilde{n}}$ satisfies (4.2) if and only if $\mathscr{P}$ satisfies

$$
\begin{aligned}
\mathscr{P}=\tilde{\Phi}^{T}(t) \mathscr{P} \tilde{\Phi}(t)+\int_{0}^{t} \tilde{\Phi}^{T}(t) \tilde{\Phi}^{-T}(s) \\
\cdot\left[\Omega\left(\mathscr{P}, B_{c}, C_{c}\right)-\sum_{i=1}^{p} \hat{\sigma}_{i}(t)\left(\tilde{A}_{i}^{T} \mathscr{P}+\mathscr{P} \tilde{A}_{i}\right)\right] \tilde{\Phi}^{-1}(s) \tilde{\Phi}(t) d s, \\
\hat{\sigma}(\cdot) \in L_{\infty}([0, \infty), \Delta), \quad t \in[0, \infty) .
\end{aligned}
$$

Proof. Suppose $\mathscr{P}$ satisfies (4.2). Then for $t \in[0, \infty)$,

$$
\begin{aligned}
0= & \tilde{\Phi}^{-T}(t)\left(\tilde{A}+\sum_{i=1}^{p} \hat{\sigma}_{i}(t) \tilde{A}_{i}\right)^{T} \mathscr{P} \tilde{\Phi}^{-1}(t)+\tilde{\Phi}^{-T}(t) \mathscr{P}\left(\tilde{A}+\sum_{i=1}^{p} \hat{\sigma}_{i}(t) \tilde{A}_{i}\right) \tilde{\Phi}^{-1}(t) \\
& +\tilde{\Phi}^{-T}(t)\left[\Omega\left(\mathscr{P}, B_{c}, C_{c}\right)-\sum_{i=1}^{p} \hat{\sigma}_{i}(t)\left(\tilde{A}_{i}^{T} \mathscr{P}+\mathscr{P} \tilde{A}_{i}\right)\right] \tilde{\Phi}^{-1}(t) \\
= & -\frac{d}{d t}\left[\tilde{\Phi}^{-T}(t) \mathscr{P} \tilde{\Phi}^{-1}(t)\right]+\tilde{\Phi}^{-T}(t)\left[\Omega\left(\mathscr{P}, B_{c}, C_{c}\right)-\sum_{i=1}^{p} \hat{\sigma}_{i}(t)\left(\tilde{A}_{i}^{T} \mathscr{P}+\mathscr{P} \tilde{A}_{i}\right)\right] \tilde{\Phi}^{-1}(t),
\end{aligned}
$$

which yields

$$
\begin{aligned}
0= & -\tilde{\Phi}^{-T}(t) \mathscr{P} \tilde{\Phi}^{-1}(t)+\mathscr{P} \\
& +\int_{0}^{t} \tilde{\Phi}^{-T}(s)\left[\Omega\left(\mathscr{P}, B_{c}, C_{c}\right)-\sum_{i=1}^{p} \hat{\sigma}_{i}(s)\left(\tilde{A}_{i}^{T} \mathscr{P}+\mathscr{P} \tilde{A}_{i}\right)\right] \tilde{\Phi}^{-1}(s) d s .
\end{aligned}
$$


Thus (4.9) is satisfied. Conversely, suppose $\mathscr{P}$ satisfies (4.9). Differentiating with respect to $t$ using Leibniz's rule yields

$$
\begin{aligned}
& 0=\left(\tilde{A}+\sum_{i=1}^{p} \hat{\sigma}_{i}(t) \tilde{A}_{i}\right)^{T} \tilde{\Phi}^{T}(t) \mathscr{P} \tilde{\Phi}(t)+\tilde{\Phi}^{T}(t) \mathscr{P} \tilde{\Phi}(t)\left(\tilde{A}+\sum_{i=1}^{p} \hat{\sigma}_{i}(t) \tilde{A}_{i}\right) \\
& +\left(\tilde{A}+\sum_{i=1}^{p} \hat{\sigma}_{i}(t) \tilde{A}_{i}\right)^{T} \int_{0}^{t} \tilde{\Phi}^{T}(t) \tilde{\Phi}^{-T}(s) \\
& \cdot\left[\Omega\left(\mathscr{P}, B_{c}, C_{c}\right)-\sum_{i=1}^{p} \hat{\sigma}_{i}(s)\left(\tilde{A}_{i}^{T} \mathscr{P}+\mathscr{P} \tilde{A}_{i}\right)\right] \tilde{\Phi}^{-1}(s) \tilde{\Phi}(t) d s \\
& +\int_{0}^{t} \tilde{\Phi}^{T}(t) \tilde{\Phi}^{-T}(s)\left[\Omega\left(\mathscr{P}, B_{c}, C_{c}\right)-\sum_{i=1}^{p} \hat{\sigma}_{i}(s)\left(\tilde{A}_{i}^{T} \mathscr{P}+\mathscr{P} \tilde{A}_{i}\right)\right] \\
& \cdot \tilde{\Phi}^{-1}(s) \tilde{\Phi}(t) d s\left(\tilde{A}+\sum_{i=1}^{p} \hat{\sigma}_{i}(t) \tilde{A}_{i}\right) \\
& +\Omega\left(\mathscr{P}, B_{c}, C_{c}\right)-\sum_{i=1}^{p} \hat{\sigma}_{i}(t)\left(\tilde{A}_{i}^{T} \mathscr{P}+\mathscr{P} \tilde{A}_{i}\right) \\
& =\left(\tilde{A}+\sum_{i=1}^{p} \hat{\sigma}_{i}(t) \tilde{A}_{i}\right)^{T} \mathscr{P}+\mathscr{P}\left(\tilde{A}+\sum_{i=1}^{p} \hat{\sigma}_{i}(t) \tilde{A}_{i}\right)+\Omega\left(\mathscr{P}, B_{c}, C_{c}\right)-\sum_{i=1}^{p} \hat{\sigma}_{i}(t)\left(\tilde{A}_{i}^{T} \mathscr{P}+\mathscr{P} \tilde{A}_{i}\right) \\
& =\tilde{A}^{T} \mathscr{P}+\mathscr{P} \tilde{A}+\Omega\left(\mathscr{P}, B_{c}, C_{c}\right) .
\end{aligned}
$$

Hence (4.2) is satisfied.

Remark 4.3. Note the identity

$$
\begin{aligned}
& \operatorname{tr} \int_{0}^{t} \tilde{\Phi}(t) \tilde{\Phi}^{-1}(s) \tilde{V} \tilde{\Phi}^{-T}(s) \tilde{\Phi}^{T}(t) d s \tilde{R}=\operatorname{tr} \int_{0}^{t} \tilde{\Phi}^{T}(t) \tilde{\Phi}^{-T}(s) \tilde{R} \tilde{\Phi}^{-1}(s) \tilde{\Phi}(t) d s \tilde{V}, \\
& \left(A_{c}, B_{c}, C_{c}\right) \in \mathbb{R}^{n_{c} \times n_{c}} \times \mathbb{R}^{n_{c} \times l} \times \mathbb{R}^{m \times n_{c}}, \quad \hat{\sigma}(\cdot) \in L_{\infty}([0, \infty), \Delta), \quad t \in[0, \infty) .
\end{aligned}
$$

We are now in a position to bound the cost $J$ by means of the matrix $\mathscr{P}$.

THEOREM 4.2. Let $\Omega: \mathbb{P}^{\tilde{n}} \times \mathbb{R}^{n_{c} \times l} \times \mathbb{R}^{m \times n_{c}} \rightarrow \mathbb{S}^{\tilde{n}}$ satisfy (4.1) and

$$
\begin{aligned}
& \sum_{i=1}^{p} \sigma_{i}\left(\tilde{A}_{i}^{T} \mathscr{P}+\mathscr{P} \tilde{A}_{i}\right)+\tilde{R} \leqq \Omega\left(\mathscr{P}, B_{c}, C_{c}\right), \quad \sigma \in \Delta, \\
& \left(\mathscr{P}, B_{c}, C_{c}\right) \in \mathbb{P}^{\tilde{n}} \times \mathbb{R}^{n_{c} \times I} \times \mathbb{R}^{m \times n_{c} .}
\end{aligned}
$$

If, for some $\left(A_{c}, B_{c}, C_{c}\right) \in \mathbb{R}^{n_{c} \times n_{c}} \times \mathbb{R}^{n_{c} \times l} \times \mathbb{R}^{m \times n_{c}}$, there exists $\mathscr{P} \in \mathbb{P}^{\tilde{n}}$ satisfying (4.2), then

$$
J\left(A_{c}, B_{c}, C_{c}\right) \leqq \operatorname{tr} \mathscr{P} \tilde{V} .
$$

Proof. From (4.8)-(4.10) and (4.3) it follows that

$$
\begin{aligned}
& J\left(A_{c}, B_{c}, C_{c}\right) \\
& =\sup _{\hat{\sigma}(\cdot) \in L_{\infty}([0, \infty), \Delta)} \limsup _{t \rightarrow \infty} \operatorname{tr}\left\{\tilde{\Phi}(t) \tilde{Q}(0) \tilde{\Phi}^{T}(t) \tilde{R}+\mathscr{P} \tilde{V}-\tilde{\Phi}^{T}(t) \mathscr{P} \tilde{\Phi}(t) \tilde{V}\right. \\
& \left.\quad-\int_{0}^{t} \tilde{\Phi}^{T}(t) \tilde{\Phi}^{-T}(s)\left[\Omega\left(\mathscr{P}, B_{c}, C_{c}\right)-\tilde{R}-\sum_{i=1}^{p} \hat{\sigma}_{i}(s)\left(\tilde{A}_{i}^{T} \mathscr{P}+\mathscr{P} \tilde{A}_{i}\right)\right] \tilde{\Phi}^{-1}(s) \tilde{\Phi}(t) d s \tilde{V}\right\} \\
& \leqq \\
& \sup _{\hat{\sigma}(\cdot) \in L_{\infty}([0, \infty), \Delta)} \limsup _{t \rightarrow \infty} \operatorname{tr}\left[\tilde{\Phi}(t) \tilde{Q}(0) \tilde{\Phi}^{T}(t) \tilde{R}+\mathscr{P} \tilde{V}\right] \\
& =\operatorname{tr} \mathscr{P} \tilde{V} .
\end{aligned}
$$


Remark 4.4. Note that since $\tilde{R} \geqq 0,(4.11)$ implies

$$
\sum_{i=1}^{p} \sigma_{i}\left(\tilde{A}_{i}^{T} \mathscr{P}+\mathscr{P} \tilde{A}_{i}\right) \leqq \Omega\left(\mathscr{P}, B_{c}, C_{c}\right), \quad \sigma \in \Delta,
$$

which is a weak form of (4.1). If $\tilde{R}>0$ then (4.11) implies (4.1). This implication is not surprising since (4.11) implies robust performance while (4.1) implies robust stability.

5. Choice of bounds. To satisfy $(4.11), \Omega(\cdot, \cdot, \cdot)$ is chosen to be of the form

$$
\Omega\left(\mathscr{P}, B_{c}, C_{c}\right)=\sum_{i=1}^{p} \Lambda_{i}\left(\mathscr{P}, B_{c}, C_{c}\right)+\tilde{R}
$$

where, for each $i=1, \cdots, p, \quad \Lambda_{i}: \mathbb{P}^{\tilde{n}} \times \mathbb{R}^{n_{\mathrm{c}} \times l} \times \mathbb{R}^{m \times n_{\mathrm{c}} \rightarrow \mathbb{S}^{\tilde{n}} \text { satisfies }}$

$$
\begin{aligned}
& \sigma_{i}\left(\tilde{A}_{i}^{T} \mathscr{P}+\mathscr{P} \tilde{A}_{i}\right) \leqq \Lambda_{i}\left(\mathscr{P}, B_{c}, C_{c}\right), \quad \sigma_{i} \in\left[-\delta_{i}, \delta_{i}\right], \\
& \left(\mathscr{P}, B_{c}, C_{c}\right) \in \mathbb{P}^{\tilde{n}} \times \mathbb{R}^{n_{c} \times l} \times \mathbb{R}^{m \times n_{c}} .
\end{aligned}
$$

Two distinct choices for the bound $\Lambda_{i}$ are considered. As we pointed out in $\S 1$, the first choice corresponds to a right shift/multiplicative white noise model [33], while the second bound generalizes results found in [26].

Proposition 5.1. For all $\alpha_{i}>0$ the function

$$
\Lambda_{i}\left(\mathscr{P}, B_{c}, C_{c}\right)=\delta_{i}\left(\alpha_{i} \mathscr{P}+\alpha_{i}^{-1} \tilde{A}_{i}^{T} \mathscr{P} \tilde{A}_{i}\right)
$$

satisfies (5.2).

Proof. Note that

$$
\begin{aligned}
0 & \leqq \\
& \left.=\sigma_{i}\left(\alpha_{i} / \delta_{i}\right)^{1 / 2} I_{\tilde{n}}-\left(\delta_{i}\right) \mathscr{P}+\left(\delta_{i} / \alpha_{i}\right)^{1 / 2} \tilde{A}_{i} \tilde{A}_{i}^{T}\right]^{T} \mathscr{P} \tilde{A}_{i}-\sigma_{i}\left(\sigma_{i}\left(\alpha_{i} / \delta_{i}\right)^{1 / 2} I_{\tilde{n}}-\left(\delta_{i} / \alpha_{i}\right)^{1 / 2} \tilde{A}_{i}\right),
\end{aligned}
$$

which, since $\sigma_{i}^{2} \leqq \delta_{i}^{2}$, implies (5.2).

Proposition 5.2. For all $\tilde{D}_{i} \in \mathbb{R}^{\tilde{n} \times \tilde{n}_{i}}$ and $\tilde{E}_{i} \in \mathbb{R}^{\tilde{n}_{i} \times \tilde{n}}$ satisfying

$$
\tilde{A}_{i}=\tilde{D}_{i} \tilde{E}_{i},
$$

the function

$$
\Lambda_{i}\left(\mathscr{P}, B_{c}, C_{c}\right)=\delta_{i}\left(\tilde{E}_{i}^{T} \tilde{E}_{i}+\mathscr{P} \tilde{D}_{i} \tilde{D}_{i}^{T} \mathscr{P}\right)
$$

satisfies (5.2).

Proof. Note that

$$
\begin{aligned}
0 & \leqq\left[\delta_{i}^{1 / 2} \tilde{E}_{i}-\sigma_{i} \delta_{i}^{-1 / 2} \tilde{D}_{i}^{T} \mathscr{P}\right]^{T}\left[\delta_{i}^{1 / 2} \tilde{E}_{i}-\sigma_{i} \delta_{i}^{-1 / 2} \tilde{D}_{i}^{T} \mathscr{P}\right] \\
& =\delta_{i} \tilde{E}_{i}^{T} \tilde{E}_{i}+\left(\sigma_{i}^{2} / \delta_{i}\right) \mathscr{P} \tilde{D}_{i} \tilde{D}_{i}^{T} \mathscr{P}-\sigma_{i}\left(\tilde{A}_{i}^{T} \mathscr{P}+\mathscr{P} \tilde{A}_{i}\right),
\end{aligned}
$$

which implies (5.2).

6. The auxiliary minimization problem and necessary conditions for optimality. To optimize robust performance while retaining robust stability, we consider the following problem for which the cost functional is given by the bound (4.12).

6.1. Auxiliary Minimization Problem. For $i=1, \cdots, p$, let $\Lambda_{i}$ be given by either (5.3) or (5.5). Determine ( $\left.\mathscr{P}, A_{c}, B_{c}, C_{c}\right) \in \mathbb{P}^{\tilde{n}} \times \mathbb{R}^{n_{c} \times n_{c}} \times \mathbb{R}^{n_{c} \times l} \times \mathbb{R}^{m \times n_{c}}$, which minimizes

$$
\mathscr{J}\left(\mathscr{P}, A_{c}, B_{c}, C_{c}\right) \triangleq \operatorname{tr} \mathscr{P} \tilde{V}
$$


subject to

$$
\begin{gathered}
0=\tilde{A}^{T} \mathscr{P}+\mathscr{P} \tilde{A}+\sum_{i=1}^{p} \Lambda_{i}\left(\mathscr{P}, B_{c}, C_{c}\right)+\tilde{R}, \\
\sum_{i=1}^{p} \sigma_{i}\left(\tilde{A}_{i}^{T} \mathscr{P}+\mathscr{P} \tilde{A}_{i}\right)<\sum_{i=1}^{p} \Lambda_{i}\left(\mathscr{P}, B_{c}, C_{c}\right)+\tilde{R}, \quad \sigma \in \Delta .
\end{gathered}
$$

Remark 6.1. Note that (6.3) enforces both (4.1) and (4.11) to guarantee robust stability and performance.

To derive first-order necessary conditions for the Auxiliary Minimization Problem, note that the constraint (6.3) defines an open set.

Proposition 6.1. The set of $\left(\mathscr{P}, B_{c}, C_{c}\right) \in \mathbb{P}^{\tilde{n}} \times \mathbb{R}^{n_{c} \times l} \times \mathbb{R}^{m \times n_{c}}$ satisfying (6.3) is open.

Proof. Since $\Lambda_{i}(\cdot, \cdot, \cdot)$ is continuous it can be shown that the function

$$
f\left(\mathscr{P}, B_{c}, C_{c}\right) \triangleq \min _{\sigma \in \Delta} \lambda_{\min }\left\{\sum_{i=1}^{p} \Lambda_{i}\left(\mathscr{P}, B_{c}, C_{c}\right)+\tilde{R}-\sum_{i=1}^{p} \sigma_{i}\left(\tilde{A}_{i}^{T} \mathscr{P}+\mathscr{P} \tilde{A}_{i}\right)\right\}
$$

is also continuous. Since (6.3) is equivalent to $0<f\left(\mathscr{P}, B_{c}, C_{c}\right)$, the result is immediate.

To obtain explicit feedback gain expressions we shall require two additional technical assumptions. If bound (5.3) is chosen for a given $i \in\{1, \cdots, p\}$ we require

$$
B_{i} \neq 0 \Rightarrow C_{i}=0 \text {, }
$$

i.e., $B_{i}$ and $C_{i}$ are not simultaneously nonzero. Of course, both $B_{i}$ and $C_{i}$ may be zero. Assumption (6.4) implies that parameter uncertainties in $B$ and $C$ must be modeled as uncorrelated. Correlation between uncertainties in $A$ and $B$ or $A$ and $C$ is, of course, permitted. Furthermore, if bound (5.5) is chosen for a given $i \in\{1, \cdots, p\}$ we require

$$
C_{i}=0 \text {. }
$$

We stress that (6.4) and (6.6) can be removed, but at the expense of explicit gain expressions.

When we use bound (5.3) the positive constant $\alpha_{i}$ will be considered fixed but arbitrary. Furthermore, for bound (5.5), let $D_{i} \in \mathbb{R}^{n \times n_{i}}, E_{i} \in \mathbb{R}^{n_{i} \times n}, H_{i} \in \mathbb{R}^{n \times m_{i}}$, and $K_{i} \in$ $\mathbb{R}^{m_{i} \times m}$ satisfy

$$
A_{i}=D_{i} E_{i}, \quad B_{i}=H_{i} K_{i},
$$

and define $\tilde{D}_{i}, \tilde{E}_{i}$ satisfying $(5.4)$ by

$$
\tilde{D}_{i} \triangleq\left[\begin{array}{cc}
D_{i} & H_{i} \\
0_{n_{c} \times n_{i}} & 0_{n_{c} \times m_{i}}
\end{array}\right], \quad \tilde{E}_{i} \triangleq\left[\begin{array}{cc}
E_{i} & 0_{n_{i} \times n_{c}} \\
0_{m_{i} \times n} & K_{i} C_{c}
\end{array}\right] .
$$

In addition to the open set defined by (6.3), the derivation of the necessary conditions requires that $\left(\mathscr{P}, A_{c}, B_{c}, C_{c}\right)$ be further restricted so that

$$
\begin{aligned}
\left(\tilde{A}+\frac{1}{2} \sum^{\prime} \delta_{i} \alpha_{i} I_{\tilde{n}}\right. & \left.+\sum^{\prime \prime} \delta_{i} \tilde{D}_{i} \tilde{D}_{i}^{T} \mathscr{P}\right) \oplus\left(\tilde{A}+\frac{1}{2} \sum^{\prime} \delta_{i} \alpha_{i} I_{\tilde{n}}+\sum^{\prime \prime} \delta_{i} \tilde{D}_{i} \tilde{D}_{i}^{T} \mathscr{P}\right) \\
& +\sum^{\prime}\left(\delta_{i} \alpha_{i}^{-1}\right) \tilde{A}_{i} \otimes \tilde{A}_{i} \text { is asymptotically stable, }
\end{aligned}
$$

In (6.8) the notation $\Sigma^{\prime}$ and $\Sigma^{\prime \prime}$ denotes summation over indices for which bounds (5.3) and (5.5), respectively, have been chosen. Note that (6.8) and (6.9) play no role in the Auxiliary Minimization Problem and thus need not be verified for robust stability or robust performance. 
For arbitrary $Q, P, \hat{Q}, \hat{P} \in \mathbb{R}^{n \times n}$ define the following notation:

$$
\begin{aligned}
& R_{2 a} \triangleq R_{2}+\sum^{\prime}\left(\delta_{i} \alpha_{i}^{-1}\right) B_{i}^{T}(P+\hat{P}) B_{i}+\sum^{\prime \prime} \delta_{i} K_{i}^{T} K_{i}, \\
& V_{2 a} \triangleq V_{2}+\sum^{\prime}\left(\delta_{i} \alpha_{i}^{-1}\right) C_{i}(Q+\hat{Q}) C_{i}^{T}, \\
& P_{a} \triangleq B^{T} P+R_{12}^{T}+\sum^{\prime}\left(\delta_{i} \alpha_{i}^{-1}\right) B_{i}^{T}(P+\hat{P}) A_{i}, \\
& Q_{a} \triangleq Q C^{T}+V_{12}+\sum^{\prime}\left(\delta_{i} \alpha_{i}^{-1}\right) A_{i}(Q+\hat{Q}) C_{i}^{T}, \\
& D \triangleq \sum^{\prime \prime} \delta_{i}\left(D_{i} D_{i}^{T}+H_{i} H_{i}^{T}\right), \quad E \triangleq \sum^{\prime \prime} \delta_{i} E_{i}^{T} E_{i}, \\
& \hat{A} \triangleq A+\frac{1}{2} \sum^{\prime} \delta_{i} \alpha_{i} I_{n}, \quad \hat{A}_{P} \triangleq \hat{A}-B R_{2 a}^{-1} P_{a}, \quad \hat{A}_{Q} \triangleq \hat{A}-Q_{a} V_{2 a}^{-1} C .
\end{aligned}
$$

The following lemma will be needed.

Lemma 6.1. If $\hat{Q}, \hat{P} \in \mathbb{N}^{n}$ and rank $\hat{Q} \hat{P}=n_{c}$, then there exist $G, \Gamma \in \mathbb{R}^{n_{c} \times n}$ and invertible $M \in \mathbb{R}^{n_{c} \times n_{c}}$ such that

$$
\begin{aligned}
& \hat{Q} \hat{P}=G^{T} M \Gamma, \\
& \Gamma G^{T}=I_{n_{c}} .
\end{aligned}
$$

Furthermore, $G, M$, and $\Gamma$ are unique except for a change of basis in $\mathbb{R}^{n}$.

Proof. The result is an immediate consequence of [45, Thm. 6.2.5, p. 123].

Note that because of (6.11), the $n \times n$ matrix $\tau \triangleq G^{T} \Gamma$ is idempotent, i.e., $\tau^{2}=\tau$. Since $\tau$ is not necessarily symmetric, it is an oblique projection. Also, define $\tau_{\perp} \triangleq I_{n}-\tau$.

Theorem 6.1. Suppose ( $\mathscr{P}, A_{c}, B_{c}, C_{c}$ ) solves the Auxiliary Minimization Problem subject to (6.8) and (6.9). Then there exist $P, Q, \hat{P}, \hat{Q} \in \mathbb{N}^{n}$ such that $\mathscr{P}, A_{c}, B_{c}, C_{c}$ are given by

$$
\begin{aligned}
\mathscr{P} & =\left[\begin{array}{cc}
P+\hat{P} & -\hat{P} G^{T} \\
-G \hat{P} & G \hat{P} G^{T}
\end{array}\right], \\
A_{c} & =\Gamma\left(A-Q_{a} V_{2 a}^{-1} C-B R_{2 a}^{-1} P_{a}+D P\right) G^{T}, \\
B_{c} & =\Gamma Q_{a} V_{2 a}^{-1}, \\
C_{c} & =-R_{2 a}^{-1} P_{a} G^{T},
\end{aligned}
$$

and such that $P, Q, \hat{P}, \hat{Q}$ satisfy

$$
\begin{aligned}
& 0=\hat{A}^{T} P+P \hat{A}+R_{1}+\sum^{\prime}\left(\delta_{i} \alpha_{i}^{-1}\right)\left[A_{i}^{T} P A_{i}+\left(A_{i}-Q_{a} V_{2 a}^{-1} C_{i}\right)^{T} \hat{P}\left(A_{i}-Q_{a} V_{2 a}^{-1} C_{i}\right)\right] \\
& +E+P D P-P_{a}^{T} R_{2 a}^{-1} P_{a}+\tau_{\perp}^{T} P_{a}^{T} R_{2 a}^{-1} P_{a} \tau_{\perp}, \\
& 0=[\hat{A}+D(P+\hat{P})] Q+Q[\hat{A}+D(P+\hat{P})]^{T}+V_{1} \\
& +\sum^{\prime \prime}\left(\delta_{i} \alpha_{i}^{-1}\right)\left[A_{i} Q A_{i}^{T}+\left(A_{i}-B_{i} R_{2 a}^{-1} P_{a}\right) \hat{Q}\left(A_{i}-B_{i} R_{2 a}^{-1} P_{a}\right)^{T}\right] \\
& -Q_{a} V_{2 a}^{-1} Q_{a}^{T}+\tau_{\perp} Q_{a} V_{2 a}^{-1} Q_{a}^{T} \tau_{\perp}^{T}, \\
& 0=\left(\hat{A}_{Q}+D P\right)^{T} \hat{P}+\hat{P}\left(\hat{A}_{Q}+D P\right)+\hat{P} D \hat{P}+P_{a}^{T} R_{2 a}^{-1} P_{a}-\tau_{\perp}^{T} P_{a}^{T} R_{2 a}^{-1} P_{a} \tau_{\perp}, \\
& 0=\left(\hat{A}_{P}+D P\right) \hat{Q}+\hat{Q}\left(\hat{A}_{P}+D P\right)^{T}+Q_{a} V_{2 a}^{-1} Q_{a}^{T}-\tau_{\perp} Q_{a} V_{2 a}^{-1} Q_{a}^{T} \tau_{\perp}^{T}, \\
& \operatorname{rank} \hat{Q}=\operatorname{rank} \hat{P}=\operatorname{rank} \hat{Q} \hat{P}=n_{c} .
\end{aligned}
$$


Conversely, if there exist $P, Q, \hat{P}, \hat{Q} \in \mathbb{N}^{n}$ satisfying (6.16)-(6.20), then $\mathscr{P}$ given by (6.12) satisfies (6.2) or, equivalently, (4.2) with $\left(A_{c}, B_{c}, C_{c}\right)$ given by (6.13)-(6.15).

Outline of proof. As discussed in $\S 1$, we limit the presentation of the proof to the salient details. First note that with the choice of bounds $\Lambda_{i},(6.2)$ becomes

$$
\begin{aligned}
0=(\tilde{A} & \left.+\frac{1}{2} \sum^{\prime} \delta_{i} \alpha_{i} I_{\tilde{n}}\right)^{T} \mathscr{P}+\mathscr{P}\left(\tilde{A}+\frac{1}{2} \sum^{\prime} \delta_{i} \alpha_{i} I_{\tilde{n}}\right)+\tilde{R} \\
& +\sum^{\prime}\left(\delta_{i} \alpha_{i}^{-1}\right) \tilde{A}_{i}^{T} \mathscr{P} \tilde{A}_{i}+\sum^{\prime \prime} \delta_{i}\left(\tilde{E}_{i}^{T} \tilde{E}_{i}+\mathscr{P} \tilde{D}_{i} \tilde{D}_{i}^{T} \mathscr{P}\right) .
\end{aligned}
$$

By introducing multipliers $\lambda \in \mathbb{R}, \lambda \geqq 0$, and $\mathscr{Q} \in \mathbb{R}^{\tilde{n} \times \tilde{n}}$, a Lagrangian can be defined as

$$
\mathscr{L}\left(\mathscr{P}, A_{c}, B_{c}, C_{c}\right) \triangleq \operatorname{tr}[\lambda \mathscr{P} \tilde{V}+\mathscr{2}(\operatorname{RHS} \text { of }(6.21))]
$$

Setting $\partial \mathscr{L} / \partial \mathscr{P}=0$ and using (6.8) implies that $\lambda=1$ without loss of generality, $2 \geqq 0$, and 2 satisfies

$$
\begin{aligned}
0=(\tilde{A} & \left.+\frac{1}{2} \sum^{\prime} \delta_{i} \alpha_{i} I_{\tilde{n}}+\sum^{\prime \prime} \tilde{D}_{i} \tilde{D}_{i}^{T} \mathscr{P P}\right) \mathscr{Q}+\mathscr{Q}\left(\tilde{A}+\frac{1}{2} \sum^{\prime} \delta_{i} \alpha_{i} I_{\tilde{n}}+\sum^{\prime \prime} \tilde{D}_{i} \tilde{D}_{i}^{T} \mathscr{P}\right)^{T} \\
& +\sum^{\prime}\left(\delta_{i} \alpha_{i}^{-1}\right) \tilde{A}_{i} \mathscr{Q} \tilde{A}_{i}^{T}+\tilde{V}
\end{aligned}
$$

The remainder of the derivation is exactly parallel to the techniques utilized in [29] and [36]. Briefly, the principal steps are as follows:

Step 1. Compute $\partial \mathscr{L} / \partial A_{c}, \partial \mathscr{L} / \partial B_{c}$, and $\partial \mathscr{L} / \partial C_{c}$.

Step 2. Use (6.9) to show that the lower right $n_{c} \times n_{c}$ blocks of $\mathscr{2}$ and $\mathscr{P}$ are positive definite.

Step 3. Use $\partial \mathscr{L} / \partial A_{c}=0$ to define a projection $\tau$ and new variables $P, Q, \hat{P}, \hat{Q}, G, \Gamma$.

Step 4. Partition (6.21) and (6.23) into six equations (1)-(6) corresponding to the $n \times n, n \times n_{c}$ and $n_{c} \times n_{c}$ blocks of $\mathscr{P}$ and 2 , respectively.

Step 5. Use (2) and (3) to solve for $A_{c}$; show that (5) and (6) also yield $A_{c}$; note that with $A_{c}$ now given, (3) and (6) are superfluous and can be eliminated.

Step 6. Manipulate (1), (2), (4), and (5) to yield (6.16)-(6.19).

Step 7. Show that Steps 4-6 are reversible so that (6.16)-(6.20) are equivalent to (6.2) or, equivalently, (4.2).

By enforcing the strict inequalities $\mathscr{P}>0$ and (6.3), solutions of (6.16)-(6.20) guarantee robust stability with a robust performance bound. The following result follows from Theorem 4.1, Theorem 4.2, and the converse of Theorem 6.1.

TheOrem 6.2. Suppose there exist $P, Q, \hat{P}, \hat{Q} \in \mathbb{N}^{n}$ satisfying (6.16)-(6.20), and suppose that (6.3) and $\mathscr{P}>0$ are satisfied with $\left(\mathscr{P}, A_{c}, B_{c}, C_{c}\right)$ given by (6.12)-(6.15). Then the compensator $A_{c}, B_{c}, C_{c}$ given by (6.13)-(6.15) solves the Robust Stability Problem and the closed-loop performance (3.7) satisfies the bound

$$
J\left(A_{c}, B_{c}, C_{c}\right) \leqq \operatorname{tr} \mathscr{P} \tilde{V} .
$$

The following existence result concerns the solvability of (6.16)-(6.20). Let $n_{u}$ denote the dimension of the unstable subspace of the plant dynamics matrix $A$.

THEOREM 6.3. Assuming $n_{c} \geqq n_{u}, R_{1}>0, V_{1}>0$, suppose the nominal plant, i.e., (3.1), (3.2) with $\delta_{i}=0, i=1, \cdots, p$, is stabilizable and detectable and, in addition, is stabilizable by means of an $n_{c}$ th-order strictly proper dynamic compensator (3.3), (3.4). Then there exist $\bar{\delta}_{1}, \cdots, \bar{\delta}_{p}>0$ such that if $\delta_{i} \in\left[0, \bar{\delta}_{i}\right), i=1, \cdots, p$, then (6.16)-(6.20) have a solution $P, Q, \hat{P}, \hat{Q} \in \mathbb{N}^{n}$ for which $\left(A_{c}, B_{c}, C_{c}\right)$ given by (6.13)-(6.15) solve the robust stability problem with robust performance bound (6.24). 
Proof. From Theorem 3.1 of [37] it follows that there exists a solution to (6.16)(6.20) that stabilizes the nominal plant. By continuity there exists a neighborhood over which robust stability with performance bound (6.24) holds.

Theorem 6.3 is an existence result that guarantees solvability of the sufficiency conditions over a range of parameter uncertainties. The actual range of uncertainty that can be bounded and the conservatism of the performance bound are problem dependent. To this end we now consider a numerical example.

7. Illustrative numerical example. To demonstrate the theory above we present an illustrative numerical example. The example chosen was originally used in [2] to illustrate the lack of a guaranteed gain margin for LQG controllers. This example was also considered in [35] for a preliminary robustness study and reconsidered in [46] using $\mu$-analysis. Define the following:

$$
\begin{array}{ll}
n=n_{u}=2, & m=l=p=1, \\
A=\left[\begin{array}{ll}
1 & 1 \\
0 & 1
\end{array}\right], \quad B=\left[\begin{array}{l}
0 \\
1
\end{array}\right], \quad C=\left[\begin{array}{ll}
1 & 0
\end{array}\right], \\
A_{1}=\left[\begin{array}{ll}
0 & 0 \\
0 & 0
\end{array}\right], \quad B_{1}=\left[\begin{array}{l}
0 \\
1
\end{array}\right], \quad C_{1}=\left[\begin{array}{ll}
0 & 0
\end{array}\right], \\
R_{1}=V_{1}=\left[\begin{array}{ll}
60 & 60 \\
60 & 60
\end{array}\right], \quad R_{12}=V_{12}=\left[\begin{array}{l}
0 \\
0
\end{array}\right], \quad R_{2}=V_{2}=1 .
\end{array}
$$

Note that the system is open-loop unstable and becomes uncontrollable at $\sigma_{1}=-1$. As can be seen using root locus, a strictly proper stabilizing controller must be of at least second order. Hence we consider (6.16)-(6.20) with $n_{c}=n$ and $\tau_{\perp}=0$. Furthermore, we use bound (5.3) and thus set $D=E=0$. Using algorithms described in

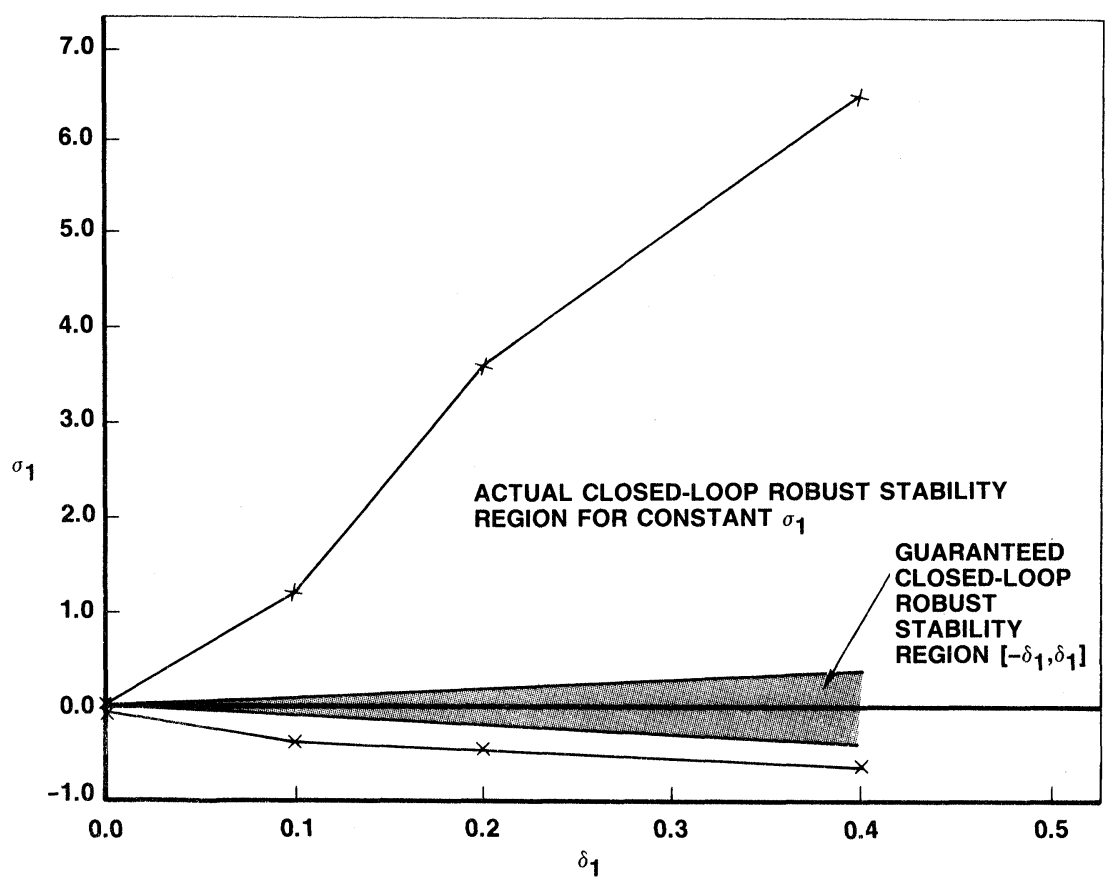

FIG. 1 


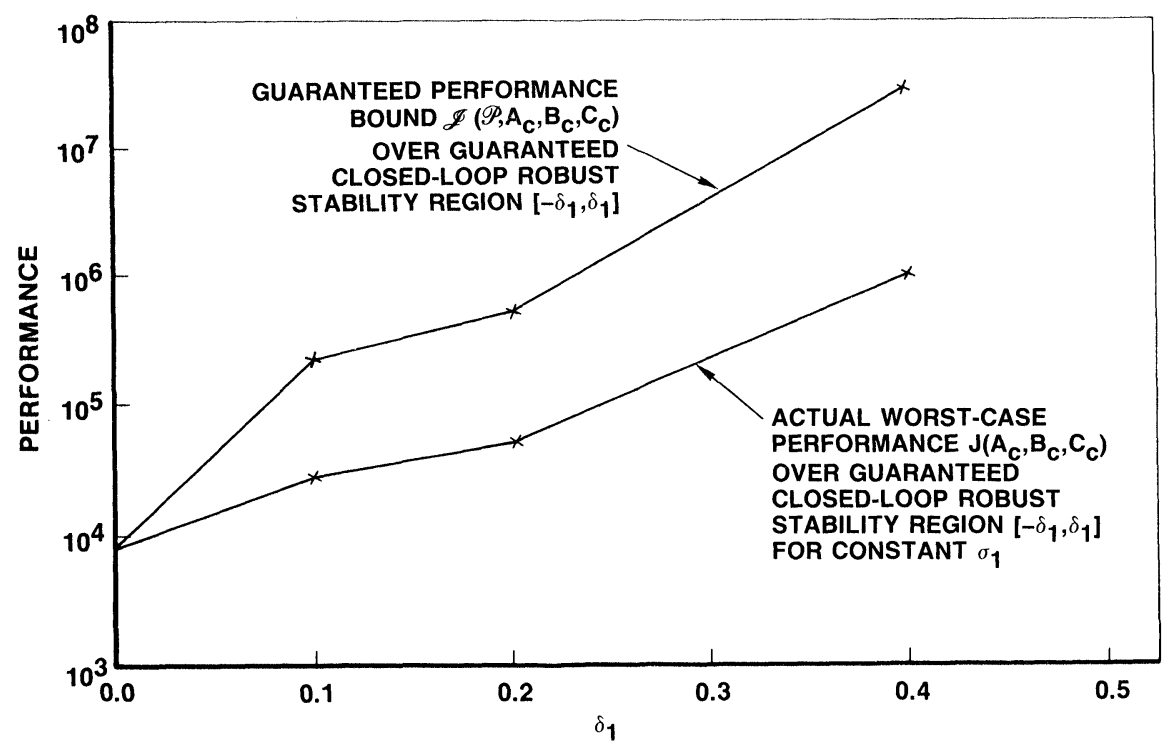

FIG. 2

TABLE 1

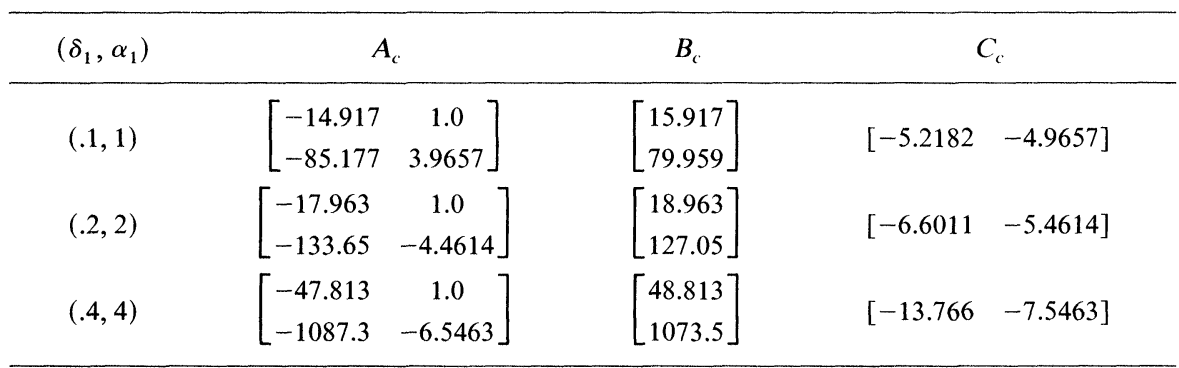

[38]-[40], controllers were obtained by solving (6.16)-(6.20) for $\left(\delta_{1}, \alpha_{1}\right)=(.1,1),(.2,2)$, and $(.4,4)$. As stated previously, these numerical solutions also verify $(4.2)$ with $\mathscr{P}$ given by (6.12). Figure 1 compares the guaranteed robust stability region to the "actual" robust stability region. This robust stability region was evaluated assuming constant $\hat{\sigma}_{1}(\cdot)$, although the theory actually guarantees robustness with respect to time-varying uncertainties. Thus, the gap between these regions may not be a reliable measure of the conservatism of the results. Note, however, that the design approach appears to provide more stability than is guaranteed a priori. This feature may be attributable to the desire for a symmetric stability interval so close to an unstabilizable plant perturbation, i.e., $\sigma_{1}=-1$. Nevertheless, the stability design objectives have been met in accordance with Theorem 6.2. Interestingly, the form of the actual stability region mimics the classical 6-dB-downward/infinite-dB-upward gain margin of full-statefeedback LQR controllers [1]. Thus, this approach appears to provide an alternative to gain-margin recovery techniques [9], which address this specialized form of plant uncertainty. Finally, Fig. 2 compares guaranteed closed-loop performance to "actual" closed-loop performance over the guaranteed closed-loop robust stability region. Again the "actual" region was determined for constant $\hat{\sigma}_{1}(\cdot)$. Controller gains are given in 
Table 1. Finally, we note that higher-order robust controllers were obtained for this example in [46] using the $\mu$-function approach.

Acknowledgments. I thank Jill M. Straehla for preparing the manuscript versions of this paper, Scott W. Greeley for carrying out the numerical computations, Dr. Wassim M. Haddad for several helpful suggestions, and the reviewers for numerous helpful comments.

\section{REFERENCES}

[1] M. G. SAFOnOV AND M. ATHANS, Gain and phase margin for multiloop LQG regulators, IEEE Trans. Automat. Control, AC-22 (1977), pp. 173-179.

[2] J. C. DOYLE, Guaranteed margins for LQG regulators, IEEE Trans. Automat. Control, AC-23 (1978), pp. 756-757.

[3] J. C. Doyle And G. Stein, Robustness with observers, IEEE Trans. Automat. Control, AC-24 (1979), pp. 607-611.

[4] - Multivariable feedback design: concepts for a classical/modern synthesis, IEEE Trans. Automat. Control, AC-26 (1981), pp. 4-16.

[5] G. ZAMES, Feedback and optimal sensitivity: model reference transformations, multiplicative seminorms, and approximate inverses, IEEE Trans. Automat. Control, AC-26 (1981), pp. 301-320.

[6] J. C. DOYLE, Analysis of feedback systems with structured uncertainties, Proc. IEEE-D, 129 (1982), pp. $242-250$.

[7] M. G. SAFONOV, Stability margins of diagonally perturbed multivariable feedback systems, Proc. IEEE-D, 129 (1982), pp. 251-256.

[8] G. ZAmes AND B. A. Francrs, Feedback, minimax sensitivity, and optimal robustness, IEEE Trans. Automat. Control, AC-28 (1983), pp. 585-601.

[9] G. STEIN AND M. ATHANS, The LQG/LTR procedure for multivariable feedback control design, IEEE Trans. Automat. Control, AC-32 (1987), pp. 105-114.

[10] B. A. Francis, A Course in $H_{\infty}$ Control Theory, Springer-Verlag, New York, 1987.

[11] E. SOROKA AND U. SHAKED, On the robustness of LQ regulators, IEEE Trans. Automat. Control, AC-29 (1984), pp. 664-665.

[12] U. SHAKED AND E. SOROKA, On the stability robustness of the continuous-time LQG optimal control, IEEE Trans. Automat. Control, AC-30 (1985), pp. 1039-1043.

[13] M. TAHK AND J. L. SPEYER, Modeling of parameter variations and asymptotic LQG synthesis, in Proc. 24th IEEE Conference on Decision and Control, Athens, Greece, December 1986, pp. 1459-1465.

[14] S. S. L. ChANG AND T. K. C. PENG, Adaptive guaranteed cost control of systems with uncertain parameters, IEEE Trans. Automat. Control, AC-17 (1972), pp. 474-483.

[15] R. V. PATEL, M. TODA, AND B. SRIDHAR, Robustness of linear quadratic state feedback designs in the presence of system uncertainty, IEEE Trans. Automat. Control, AC-22 (1977), pp. 945-949.

[16] G. LeITMANN, Guaranteed asymptotic stability for a class of uncertain linear dynamical systems, J. Optim. Theory Appl., 27 (1979), pp. 96-106.

[17] A. VinkLER AND L. J. WoOD, Multistep guaranteed cost control of linear systems with uncertain parameters, J. Guid. Control, 2 (1979), pp. 449-456.

[18] M. Eslami AND D. L. Russell, On stability with large parameter variations: stemming from the direct method of Lyapunov, IEEE Trans. Automat. Control, AC-25 (1980), pp. 1231-1234.

[19] M. CORLESS AND G. LEITMANN, Continuous state feedback guaranteeing uniform ultimate boundedness for uncertain dynamical systems, IEEE Trans. Automat. Control, AC-26 (1981), pp. 1139-1144.

[20] J. S. THORP AND B. R. BARMISH, On guaranteed stability of uncertain linear systems via linear control, J. Optim. Theory Appl., 35 (1981), pp. 559-579.

[21] B. R. BArmish, M. Corless, AND G. Leitmann, A new class of stabilizing controllers for uncertain dynamical systems, SIAM J. Control Optim., 21 (1983), pp. 246-255.

[22] B. R. BArmish, I. R. Petersen, AND A. Feuer, Linear ultimate boundedness control of uncertain dynamic systems, Automatica, 19 (1983), pp. 523-532.

[23] B. R. BARMISH, Necessary and sufficient conditions for quadratic stabilizability of an uncertain linear system, J. Optim. Theory Appl., 46 (1985), pp. 399-408.

[24] R. K. Yedavalli, S. S. BAnda, AND D. B. Ridgely, Time-domain stability robustness measures for linear regulators, J. Guid. Control Dyn., 8 (1985), pp. 520-524. 
[25] A. R. GAlimidi AND B. R. BARMish, The constrained Lyapunov problem and its application to robust output feedback stabilization, IEEE Trans. Automat. Control, AC-31 (1986), pp. 410-419.

[26] I. R. PETERSEN AND C. V. HOLlot, A Riccati equation approach to the stabilization of uncertain systems, Automatica, 22 (1986), pp. 397-411.

[27] G. Leitmann, E. P. Ryan, And A. STEInberg, Feedback control of uncertain systems: robustness with respect to neglected actuator and sensor dynamics, Internat. J. Control, 43 (1986), pp. 1243-1256.

[28] O. I. KOSMIDOU AND P. BERTRAND, Robust-controller design for systems with large parameter variations, Internat. J. Control, 45 (1987), pp. 927-938.

[29] D. C. HYLAND AND D. S. BERNSTEIN, The optimal projection equations for fixed-order dynamic compensation, IEEE Trans. Automat. Control, AC-29 (1984), pp. 1034-1037.

[30] D.S. BERNSTEIN AND D. C. HYLAND, The optimal projection equations for finite-dimensional fixed-order dynamic compensation of infinite-dimensional systems, SIAM J. Control Optim., 23 (1986), pp. 122-151.

[31] D. S. BERNSTEIN, The optimal projection equations for static and dynamic output feedback: the singular case, IEEE Trans. Automat. Control, AC-32 (1987), pp. 1139-1143.

[32] D. S. BERNSTEIN AND W. M. HADDAD, LQG control with an $H_{\infty}$ performance bound: a Riccati equation approach, IEEE Trans. Automat. Control, AC-34 (1989), to appear.

[33] D. S. BERNSTEIN, Robust static and dynamic output feedback stabilization: deterministic and stochastic perspectives, IEEE Trans. Automat. Control, AC-32 (1987), pp. 1076-1084.

[34] Y. A. PHILlis, Controller design of systems with multiplicative noise, IEEE Trans. Automat. Control, AC-30 (1985), pp. 1017-1019.

[35] D. S. BERNSTEIN AND S. W. GREELEY, Robust controller synthesis using the maximum entropy design equations, IEEE Trans. Automat. Control, AC-31 (1986), pp. 362-364.

[36] D. S. BERNSTEIN AND D. C. HYLAND, The optimal projection equations for reduced-order modelling, estimation and control of linear systems with multiplicative white noise, J. Optim. Theory Appl., 58 (1988), pp. 387-409.

[37] S. RiCHTER, A homotopy algorithm for solving the optimal projection equations for fixed-order dynamic compensation: existence, convergence and global optimality, in Proc. American Control Conference, Minneapolis, MN, June 1987, pp. 1527-1531.

[38] S. W. GREELEY AND D. C. HYLAND, Reduced-order compensation: LQG reduction versus optimal projection using a homotopic continuation method, Proc. IEEE Conference on Decision and Control, Los Angeles, CA, December 1987, pp. 742-747.

[39] A. GruzEN, Robust reduced order control of flexible structures, Report CSDL-T-900, Charles Stark Draper Laboratory, Cambridge, MA, April 1986.

[40] A. GRUZEN AND W. E. VANDER VELDE, Robust reduced-order control of flexible structures using the optimal projection/ maximum entropy design methodology, AIAA Guid. Nav. Contr. Conf., Williamsburg, VA, August 1978.

[41] J. W. BREWER, Kronecker products and matrix calculus in system theory, IEEE Trans. Circuits and Systems, CAS-25 (1978), pp. 772-781.

[42] J. K. Hale, Ordinary Differential Equations, John Wiley, New York, 1969.

[43] J. Warga, Optimal Control of Differential and Functional Equations, Academic Press, New York, 1972.

[44] L. Arnold, Stochastic Differential Equations: Theory and Applications, John Wiley, New York, 1974.

[45] C. R. Rao And S. K. Mitra, Generalized Inverse of Matrices and Its Applications, John Wiley, New York, 1971.

[46] A. Packard AND J. C. Doyle, Robust control with an $\mathrm{H}_{2}$ performance objective, in Proc. American Control Conference, Minneapolis, MN, June 1987, pp. 2141-2145. 